\title{
Multiple peripheral emboli after cardiac trauma
}

\author{
P KERTES, G WESTLAKE, M LUXTON \\ From the Departments of Cardiology and Cardiothoracic Surgery, The Royal Melbourne Hospital, Melbourne, Australia
}

SUMMARY The case is presented of a 25 year old footballer with multiple systemic emboli from a left ventricular apical thrombus which occurred as a delayed complication of non-penetrating cardiac trauma. The presentation was with intermittent claudication, and this case demonstrates the need to suspect multiple embolism in younger patients with occlusive peripheral vascular disease and to exclude a cardiac source.

\section{Case history}

A 25 year old Australian Rules footballer was referred for cardiac consultation in May 1981. He had originally presented to an orthopaedic surgeon in February 1981 with a six month history of pain in first the left and later the right foot during football matches. A diagnosis of metatarsalgia was made, and he underwent surgery to both feet. At operation it was noted that the left tibial artery was occluded. The operation was complicated by deep venous thrombosis, which was treated with a short course of anticoagulants. Exertional pain and numbness continued in both feet postoperatively and he was referred for femoral angiography and cardiological opinion.

There was no significant past medical history or family history of cardiac disease, and the patient was a non-smoker. There was no history of chest pain. Clinical examination disclosed absent pulses in both feet with normal femoral pulses and no other abnormalities in the cardiovascular system. An electrocardiogram disclosed an old inferior infarction, with pathological $Q$ waves and $T$ wave inversion in the inferior leads. Chest $x$-ray film was normal. All biochemical and haematological variables tested were within the normal range; in particular, specific testing for the presence of a hypercoagulable state was normal. Fasting serum lipids were also normal. An echocardiogram was requested to exclude left atrial myxoma, and both $M$-mode and two dimensional scans were reported as normal.

Femoral angiography was performed and showed occlusion of the left anterior and posterior tibial arteries just beyond their origins with collateral flow through the peroneal arteries, with poor peripheral reconstitution. In the right leg, extensive thrombus was noted in the anterior tibial artery, with a small thrombus also present in the profunda femoris vessel.

Despite a normal echocardiogram, it was decided to perform coronary angiography and left ventriculography. The left coronary system was completely normal but there was an extensive filling defect throughout the right coronary artery, consistent with recanalising thrombus (Fig. 1). The left ventriculogram showed mild inferior hypokinesis with normal anteroapical contraction, and a small, pedunculated, and mobile filling defect was noted at the apex of the left ventricle (Fig. 2). A provisional diagnosis of left ventricular tumour was made and surgical excision advised.

At operation, a $1 \mathrm{~cm}$ red "tumour" was found at the apex (Fig. 3), attached to the endocardium by several cord-like structures. The lesion was removed together with its endocardial base through a left ventriculotomy. Histology of the excised specimen showed organising thrombus around strands of normal myocardium, with no evidence of tumour tissue. The appearances were consistent with thrombosis secondary to ruptured trabeculae. When questioned, the patient remembered a blow to the left chest while playing football which had resulted in fractured ribs, some months before the onset of the claudication. No other history of chest trauma was obtained. The patient's postoperative recovery was excellent but a few weeks later he presented again with an acute left brachial artery occlusion requiring embolectomy. This was considered to be secondary to a thrombus at the site of the left ventriculotomy, and his subsequent progress has been uneventful. 


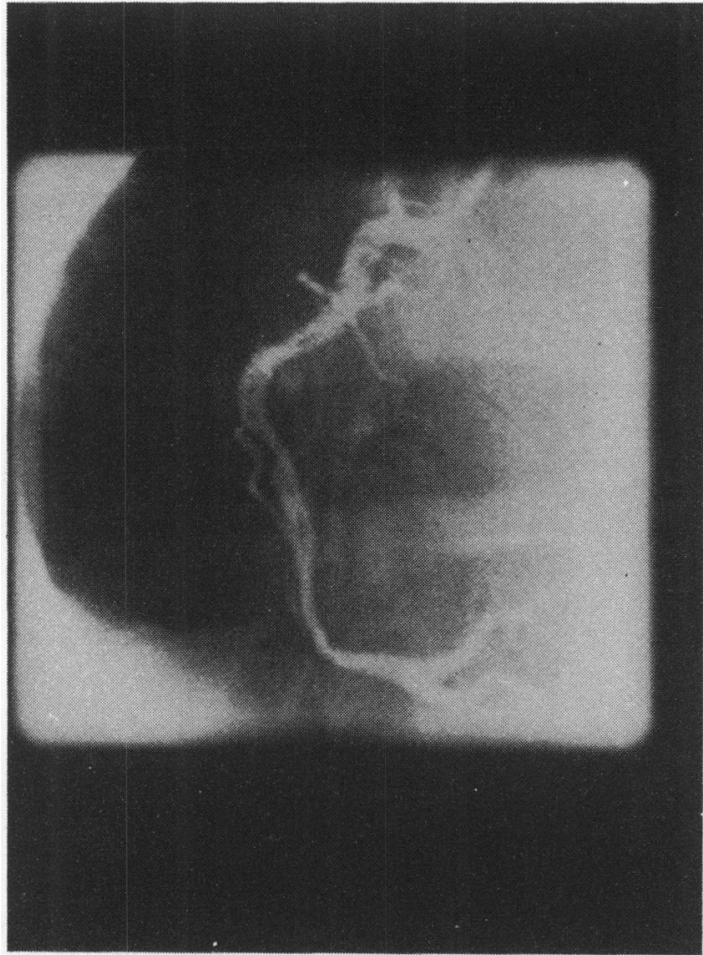

Fig. 1 Angiogram of the right coronary artery showing an extensive filling defect suggesting recanalising thrombus.

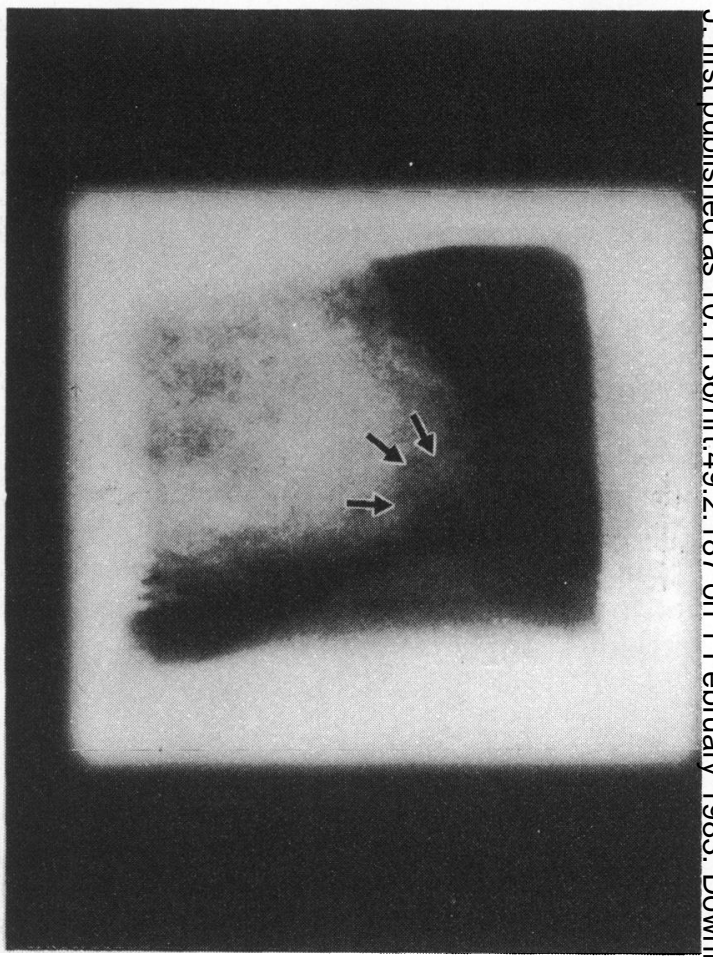

Fig. 2 Left ventricular angiogram showing a small, pedunculated lesion at the apex. 


\section{Discussion}

Peripheral circulatory disorders in young patients should suggest the possibility of recurrent embolism from the heart. Rheumatic disease is usually evident on examination and is confirmed by echocardiography. Paroxysmal atrial fibrillation is suggested by a history of palpitation, and can usually be confirmed with ambulatory monitoring or electrocardiograph telephone transmission. Mitral valve prolapse has been associated with peripheral, and particularly cerebral, embolism $^{1}$; in most cases a systolic click and/or murmur together with characteristic echocardiographic findings will establish the diagnosis. Finally, myxomas are a rare but treatable cause of systemic emboli and can usually be diagnosed on echocardiography. Though most often situated in the left atrium, left ventricular myxomas have been described. ${ }^{2}$

Cardiac injury may occur after apparently mild, nonpenetrating chest wall trauma ${ }^{3}$ and the types of injury that can occur have been extensively reviewed by Parmley and co-workers. ${ }^{4}$ Myocardial contusion is the commonest form of non-fatal injury sustained, and histology shows muscle fibre disruption and fragmentation, with secondary haemorrhage and oedema. Myocardial infarction after traumatic injury to otherwise normal coronary arteries has also been described..$^{5-7}$ Mural thrombus canoccur secondary to large areas of contusion or infarction (with or without left ventricular aneurysm), and may subsequently result in peripheral embolism. Few such cases, however, have been published. In Parmley et al.'s series, ${ }^{4}$ there were two deaths resulting from systemic embolism in their 546 necropsy cases. In a series of six patients with cardiac injury occurring after blunt chest wall trauma, Mackintosh and Fleming ${ }^{7}$ reported a case of multiple cerebral embolism from mural thrombus secondary to anterior infarction, with histologically proven traumatic occlusion of the left anterior descending artery. Kessler et al. ${ }^{8}$ have recently reported a case of pulmonary embolism caused by pedunculated right ventricular thrombus after repeated blunt chest trauma. In their case, a presumptive diagnosis of right ventricular myxoma was made on echocardiography.

We believe that in our patient, closed chest injury produced rupture of trabeculae at the apex of the left ventricle resulting in local thrombosis, with subsequent embolism to both legs and also the right coronary artery leading to inferior myocardial infarction. It is unusual that he did not present with chest pain at that time, though it is conceivable that the ischaemic pain may have been ascribed to the chest wall injury.

We have considered the alternative explanation that the primary event was traumatic injury to the right coronary artery, resulting in inferior infarction, mural thrombus, and subsequent peripheral embolism. We believe this alternative explanation is untenable for two reasons: the thrombus was situated at the apex of the left ventricle and clearly removed from the hypokinetic area seen on the left ventriculogram, and the histology of the excised specimen showed normal myocardium at the base of and within the thrombus.

This case report highlights the need to exclude carefully a cardiac source for peripheral embolism in younger patients with occlusive vascular disease, and also shows that even apparently mild blunt chest wall trauma may result in major cardiac damage. Though the first echocardiogram in our. patient did not detect the lesion, possibly because of its small size and apical position, echocardiography is a principal investigation if embolism from the heart is suspected.

\section{References}

1 Barnett HJM, Jones MW, Boughner DR, Kostuk WJ. Cerebral ischemic events associated with prolapsing mitral valve. Arch Neurol 1976; 33: 777-82.

2 Meller J, Teichholz LE, Pichard AD, et al. Left ventricular myxoma: echocardiographic diagnosis and review of the literature. Am F Med 1977; 63: 816-23.

3 Watson JH, Bartholomae WM. Cardiac injury due to nonpenetrating chest trauma. Ann Intern Med 1960; 52: 871-80.

4 Parmley LF, Manion WC, Mattingly TW. Nonpenetrating traumatic injury of the heart. Circulation 1958; 18: 371-96.

5 Oren A, Bar-Shlomo B, Stern S. Acute coronary occlusion following blunt injury to the chest in the absence of coronary atherosclerosis. Am Heart $\mathcal{f}$ 1976; 92: 501-5.

6 Harthorne JW, Kantrowitz PA, Dinsmore RE, Sanders CA. Traumatic myocardial infarction: report of a case with normal coronary angiogram. Ann Intern Med 1967; 66: $341-4$.

7 Mackintosh AF, Fleming HA. Cardiac damage presenting late after road accidents. Thorax 1981; 36: 811-3.

8 Kessler KM, Mallon SM, Bolooki H, Myerburg RJ. Pedunculated right ventricular thrombus due to repeated blunt chest trauma. Am Heart f 1981; 102: $1064-6$.

Requests for reprints to Dr M Luxton, Department of Cardiology, c/o Post Office, The Royal Melbourne Hospital, Victoria 3050, Australia. 